\title{
Evolution and colors of helium-core white dwarf stars with high-metallicity progenitors
}

\author{
L. G. Althaus ${ }^{1,4, \star}$, J. A. Panei ${ }^{1,2}$, A. D. Romero ${ }^{1,2, \star \star}$, R. D. Rohrmann ${ }^{3, \star}$, A. H. Córsico ${ }^{1,2, \star}$, \\ E. García-Berro ${ }^{4,5}$, and M. M. Miller Bertolami ${ }^{1,2, \star \star}$
}

\author{
1 Facultad de Ciencias Astronómicas y Geofísicas, Universidad Nacional de La Plata, Paseo del Bosque S/N, (B1900FWA)La Plata, \\ Argentina \\ e-mail: althaus@fcaglp.unlp.edu.ar \\ 2 Instituto de Astrofísica de La Plata, IALP (CCT La Plata), CONICET-UNLP, Argentina \\ 3 Observatorio Astronómico, Universidad Nacional de Córdoba, Laprida 854 (5000), Córdoba, Argentina \\ 4 Departament de Física Aplicada, Escola Politècnica Superior de Castelldefels, Universitat Politècnica de Catalunya, \\ Av. del Canal Olímpic, s/n, 08860 Castelldefels, Spain \\ 5 Institute for Space Studies of Catalonia, c/Gran Capità 2-4, Edif. Nexus 104, 08034 Barcelona, Spain
}

Received 9 January 2009 / Accepted 18 April 2009

\section{ABSTRACT}

\begin{abstract}
Aims. Motivated by the recent detection of single and binary He-core white dwarfs in metal-rich clusters, we present a full set of evolutionary calculations and colors appropriate to the study of these white dwarfs. The paper is also aimed at investigating whether stable hydrogen burning may constitute a major source of energy for massive He-core white dwarfs resulting from high-metallicity progenitors.

Methods. White dwarf sequences are derived by considering the evolutionary history of progenitor stars with supersolar metallicities. We also incorporate a self-consistent, time-dependent treatment of gravitational settling and chemical diffusion, as well as of the residual nuclear burning.

Results. We find that the influence of residual nuclear burning during the late stages of white dwarf evolution is strongly dependent on the chemical diffusion at the base of the hydrogen-rich envelope. When no diffusion is considered, residual hydrogen burning strongly influences the advanced stages of white dwarf cooling, introducing evolutionary delays of several Gyr. By contrast, when diffusion is taken into account, the role of residual nuclear burning is strongly mitigated, and the evolution is only dictated by the thermal content stored in the ions. In addition, for all of our sequences, we provide accurate color and magnitudes on the basis of new and improved non-gray model atmospheres that explicitly include Ly $\alpha$ quasi-molecular opacity.
\end{abstract}

Key words. stars: evolution - stars: white dwarfs - diffusion - galaxies: clusters: general

\section{Introduction}

White dwarfs constitute the most common endpoint of stellar evolution. In fact, more than $95 \%$ of all stars are expected to end their lives as white dwarfs. As such, the present population of white dwarfs conveys information about the history of our Galaxy and has potential applications as reliable cosmic clocks to infer the age of a wide variety of stellar populations - see Winget \& Kepler (2008) for a recent review.

The mass distribution of hot white dwarfs (Kepler et al. 2007) peaks at about $0.59 M_{\odot}$ and exhibits a significant lowmass tail of white dwarfs with masses lower than $0.45 M_{\odot}$, which peaks at $0.40 M_{\odot}$. These low-mass white dwarfs are the result of strong mass-loss episodes during the red giant branch (RGB) evolution before the onset of the helium core flash. For solar metallicity stars this is thought to occur only in binary systems. That low-mass He-core white dwarfs are predominantly found in close binary systems - mostly with another white dwarf or a neutron star companion (van Kerkwijk et al. 2005) - supports the idea that most of these white dwarfs are the result of mass-tranfer

* Member of the Carrera del Investigador Científico y Tecnológico, CONICET, Argentina.

$\star \star$ Fellow of CONICET, Argentina. episodes during binary evolution. Here, a companion strips the outer envelope from a post main-sequence star before the star reaches the tip of the RGB. Accordingly, and due to the relative simplicity of white dwarf evolution, He-core white dwarfs have been used to constrain the ages and properties of millisecond pulsars (van Kerkwijk et al. 2005).

The interest in low-mass white dwarfs is also motivated by the discovery of candidate He-core white dwarfs in open and globular clusters. We mention the open cluster M67 (Landsman et al. 1997), the globular cluster NGC 6397 - where the first He-core white dwarf in a globular cluster was identified (Cool et al. 1998; Taylor et al. 2001) - and 47 Tuc (Edmonds et al. 2001). More recently, it has been suggested that the excess of very hot (extreme) horizontal branch (EHB) stars in $\omega$ Cen and in NGC 2808 - which are assumed to be the result of strong massloss episodes on the RGB - could be reflecting the existence of a sizeable fraction of He-core white dwarfs in these Galactic globular clusters (Castellani et al. 2007; Calamida et al. 2008). In fact, as discussed in Calamida et al. (2008), He-core white dwarfs have been detected in stellar systems that harbor a large fraction of EHB stars, thus implying that a fraction of RGB stars might avoid the helium core flash (Castellani et al. 1994). The presence of He-core white dwarfs in these clusters is the result of extreme mass loss possibly caused by stellar encounters in the 
high stellar-density environment of globular clusters or by evolution in compact binaries. Thus, a large population of He-core white dwarfs offers the possibility of constraining globular cluster dynamics and evolution (Moehler \& Bono 2008).

The possible detection of single low-mass white dwarfs in NGC 6791 , one of the oldest ( $\gtrsim 8 \mathrm{Gyr}$ ) and most metal-rich $([\mathrm{Fe} / \mathrm{H}] \approx+0.4)$ open clusters in our Galaxy (Origlia et al. 2006), has sparked a vivid debate. Kalirai et al. (2007) have spectroscopically identified a few very bright white dwarfs in the upper part of the cooling sequence of NGC 6791. Their masses are apparently consistent with single massive He-cores, and the mean mass of these white dwarfs is $\langle M\rangle=0.43 \pm 0.06 M_{\odot}$. The existence of a large population of massive He-core white dwarfs in this extremely metal-rich cluster was suggested by Kalirai et al. (2007) on the basis that NGC 6791 contains a large number of EHB stars. These He-core white dwarfs and EHB populations would be, according to Kalirai et al. (2007), the result of enhanced mass losses on the RGB in this cluster, perhaps driven by its high metal content. However, Van Loon et al. (2008) have recently suggested that there is no strong mass loss among the stars in NGC 6791. Owing to the longer cooling times of He-core white dwarfs, a substantial fraction of massive He-core white dwarfs were invoked by Hansen (2005) to explain the observed bright peak in the white dwarf luminosity function of NGC 6791, although this has been recently challenged by Bedin et al. (2008a) who claim that the peak in the luminosity function can be accounted for by a large population of double white dwarf binary systems in the cluster. Finally, based on theoretical arguments about the initial-final mass relationship for stars of different metallicities, Meng et al. (2008) have shown that metal-rich, low-mass stars may become undermassive white dwarfs. According to these authors, a population of undermassive white dwarfs $\left(M<0.5 M_{\odot}\right)$ are expected in metal-rich old clusters.

A proper interpretation of the observations of He-core white dwarfs in high-metallicity environments like that of NGC 6791 requires evolutionary calculations. In particular, it requires computing full evolutionary sequences of white dwarfs with supersolar metallicity progenitors. However, a detailed study of the evolutionary properties of He-core white dwarfs resulting from progenitors with extreme metallicity does not exist in the literature. The present work is intended to fill this shortcoming. Specifically, we computed a full set of evolutionary calculations for He-core white dwarfs covering a wide range of stellar masses. We considered the evolutionary history of progenitor stars for the case of supersolar metallicities of $Z=0.03,0.04$, and 0.05 . The pre white dwarf evolution has been simulated by removing mass from a $1 M_{\odot}$ model at the appropriate stages of its evolution. We present evolutionary calculations that include a self-consistent and time-dependent treatment of gravitational settling and of chemical diffusion, as well as a careful study of the role played by residual nuclear burning. In particular, one of the aims of this work is to investigate whether stable hydrogen burning may constitute the main source of energy for evolved He-core white dwarfs resulting from high-metallicity progenitors. In addition, for all our sequences, we provide accurate colors and magnitudes based on new and improved non-gray model atmospheres that explicitly include $\operatorname{Ly} \alpha$ quasi-molecular opacity according to the approximation used by Kowalski \& Saumon (2006). In view of the possibility that some He-core white dwarfs in NGC 6791 could have masses higher than the canonical value of the helium core mass at the helium flash (about $0.45 M_{\odot}$ at the high metallicity of NGC 6791), we extended the mass range of our calculations computing evolutionary sequences for He-core white dwarfs with masses up to $0.55 M_{\odot}$. To this end, we artificially suppressed the occurrence of the He-core flash at the tip of the RGB. The possibility of He-core white dwarfs above $\sim 0.50 M_{\odot}$, possibly due to rotation, has been recently discussed by Bedin et al. (2008b) as an alternative to explaining some observed features of the RGB and cooling sequences in NGC 6791. This paper is organized as follows. In Sect. 2 we comment on the main existing evolutionary calculations for He-core white dwarfs. Section 3 contains details about the main ingredients of our evolutionary calculations and of the non-gray model atmosphere we use to compute the colors and magnitudes of our evolutionary sequences. In Sect. 4 we describe our results, emphasizing the role of nuclear burning and element diffusion. In this section we also discuss the evolution of massive He-core white dwarfs. In Sect. 5 we briefly comment on the predictions of our model atmospheres for the white dwarf sequences. Finally, Sect. 6 is devoted to discussing and summarizing our results.

\section{Evolutionary calculations for He-core white dwarfs}

The study of the evolution of low-mass He white dwarfs has captured the attention of researchers since Kippenhahn et al. (1967) suggested that these stars could be the natural result of substantial mass loss from low-mass red giant stars filling their Roche lobes in binary systems. Later, Webbink (1975) was the first to show that for masses greater than $0.17 M_{\odot}$, He-core white dwarfs develop hydrogen shell flashes. He found that the cooling of these white dwarfs was significantly affected by the contribution of residual proton-proton (pp) hydrogen burning in the envelope. Long cooling times resulting from residual hydrogen burning were also reported by Castellani et al. (1994). These authors considered the evolutionary history of the progenitor stars and clearly demonstrated that, for massive He-core white dwarfs (even close to the upper limit for the mass of He-core white dwarfs), the contribution of pp burning can noticeable slow down the cooling up to $\log \left(L / L_{\odot}\right) \sim-4$ and that for lower masses the cooling is progressively affected by strong CNO flashes, the occurrence of which depends on the metal content of the parent star.

Althaus \& Benvenuto (1997) and Hansen \& Phinney (1998) also presented cooling models for low-mass He-core white dwarfs. However, because these studies neglected the evolutionary history of the progenitor stars, the role of hydrogen flashes and of residual nuclear burning were not adequately addressed. Hansen \& Phinney (1998) found that thin envelopes allow He-core white dwarfs to cool rapidly, while for white dwarfs with thick envelopes residual hydrogen burning slows down the cooling. The importance of including the evolution of progenitor stars to properly address the role of residual hydrogen burning during the final cooling branch was pointed out by Driebe et al. (1998) and Sarna et al. (2000), who reported several hydrogen shell flashes during the cooling phase. Most important, the evolutionary calculations of these authors resulted in models with thick envelopes in all cases, including those that experience thermonuclear flashes and, hence, they derived long white dwarf cooling timescales due to residual hydrogen burning. The resulting ages are thus much greater than those resulting from considering only the decrease in the thermal content of the ions. More recently, Nelson et al. (2004) have also found that hydrogen burning strongly delays the cooling process, especially for very low-mass white dwarfs. They found flashes taking place for masses in the range $0.21 M_{\odot} \lesssim M_{\mathrm{WD}} \lesssim 0.28 M_{\odot}$, in good agreement with the predictions of Driebe et al. (1998). 
The studies mentioned above show that residual hydrogen burning may constitute the main energy source for He-core white dwarfs, even for massive ones, a key aspect in interpretating of the observational characteristics of these stars (Driebe at al. 1998). However, the role of hydrogen burning may be strongly mitigated by element diffusion. Indeed, Iben \& Tutukov (1986) showed that chemical diffusion leads to additional CNO flashes related to diffusion of hydrogen inward, where hotter material is present. These authors concluded that the occurrence of similar flashes would further reduce the H-rich envelope, eventually becoming too small to sustain any further nuclear burning. The importance of element diffusion in inducing additional CNO flashes was demonstrated by Althaus et al. (2001a,b), who found that element diffusion strongly affects the structure and cooling history of He-core white dwarfs. Indeed, these authors found that diffusion-induced CNO flashes yield small hydrogen envelopes and, thus, evolution is fast at late stages. Althaus et al. (2001a,b) found that diffusion is a key physical ingredient in explaining the age dichotomy suggested by observations of those He-core white dwarfs that are companions to millisecond pulsars (Bassa et al. 2003; Bassa 2006). In addition, Althaus et al. (2001a) found that the mass range for CNO flashes strongly depends on the inclusion of diffusion processes in evolutionary calculations.

The importance of diffusion in He-core white dwarfs with progenitors having supersolar metallicities has not been addressed so far in the literature. However, this is an important issue that deserves further study since it has relevant consequences for the age determinations of stellar clusters. The evolutionary calculations to be presented here, together with those we presented in Serenelli et al. (2002) for He-core white dwarfs of lowmetallicity progenitors constitute a solid and consistent frame for interpreting observations of He-core white dwarfs in stellar clusters having a wide range of metallicities.

\section{Computational details}

As previously stated, one of the aims of this work is to provide a set of white dwarf cooling tracks (including ages, colors, and magnitudes) appropriate for studying low-mass He-core white dwarfs in metal-rich environments. This grid of models covers a wide range of stellar masses as shown in Table 1. Three supersolar metallicities for the progenitor star have been considered: $Z=0.03,0.04$, and 0.05 . Specifically, we followed the evolution of 30 white dwarf sequences from the end of the massloss episode during the pre-white dwarf evolution down to very low surface luminosities. The initial He content at the main sequence is given by $Y=0.23+2.4 Z$, which is consistent with present determinations of the chemical evolution of the Galaxy (Flynn 2004; Casagrande et al. 2007). Thus, the initial compositions of our sequences are, respectively, $(Y, Z)=(0.302,0.03)$, $(Y, Z)=(0.326,0.04)$, and $(Y, Z)=(0.35,0.05)$. To study the evolution of He-core white dwarfs with masses higher than the helium core mass at the helium flash, we also computed some white dwarf sequences artificially suppressing the core helium flash at the tip of the RGB. Additional sequences without diffusion and also without nuclear burning were also computed to assess the impact of nuclear burning and diffusion processes on the evolutionary properties of white dwarfs. This allows exploration of the role played by diffusion in the occurrence of additional hydrogen shell flashes in He-core white dwarfs and, more important, to investigate whether or not the hydrogen envelope mass can be considerably reduced by enhanced hydrogen burning during these flash episodes. The evolutionary stages prior to
Table 1. Selected properties of our He-core white dwarf sequences at the point of maximum effective temperature: metallicity of progenitor star, stellar mass, mass of hydrogen in the outer layers, $M_{\mathrm{H}}$, and surface hydrogen abundance by mass.

\begin{tabular}{cccc}
\hline \hline$Z$ & $M / M_{\odot}$ & $M_{\mathrm{H}} / M_{\odot}\left[10^{-3}\right]$ & $X_{\mathrm{H}}$ \\
\hline 0.03 & 0.220 & 2.07 & 0.490 \\
& 0.250 & 1.51 & 0.531 \\
& 0.303 & 0.98 & 0.658 \\
& 0.358 & 0.56 & 0.658 \\
& 0.400 & 0.40 & 0.658 \\
& 0.452 & 0.27 & 0.658 \\
& $0.513^{\dagger}$ & 0.20 & 0.658 \\
& $0.522^{\dagger}$ & 0.17 & 0.658 \\
\hline 0.04 & 0.212 & 1.96 & 0.464 \\
& 0.272 & 1.17 & 0.622 \\
& 0.283 & 1.04 & 0.622 \\
& 0.289 & 0.98 & 0.622 \\
& 0.299 & 0.88 & 0.622 \\
& 0.306 & 0.81 & 0.622 \\
& 0.319 & 0.72 & 0.621 \\
& 0.337 & 0.60 & 0.622 \\
& 0.365 & 0.48 & 0.625 \\
& 0.403 & 0.35 & 0.623 \\
& 0.445 & 0.26 & 0.617 \\
\hline 0.05 & 0.207 & 2.26 & 0.430 \\
& 0.273 & 1.06 & 0.588 \\
& 0.288 & 0.91 & 0.588 \\
& 0.295 & 0.84 & 0.588 \\
& 0.301 & 0.78 & 0.588 \\
& 0.311 & 0.72 & 0.588 \\
& 0.323 & 0.63 & 0.588 \\
& 0.348 & 0.51 & 0.588 \\
& 0.368 & 0.43 & 0.585 \\
& 0.401 & 0.34 & 0.588 \\
0.445 & 0.25 & 0.588 \\
\hline & & & \\
& & & \\
& & & \\
& &
\end{tabular}

$\dagger$ These sequences were obtained by artificially suppressing the core helium flash at the RGB. They are discussed in Sect. 4.3.

the formation of the white dwarf are accounted for by computing the evolution of a $1 M_{\odot}$ model star for the three mentioned metallicities from the main sequence to the red giant stage (see later in this section). In what follows, we comment on the input physics considered in our calculations, the initial models, and our atmospheric treatment.

\subsection{Evolutionary code and input physics}

The calculations presented in this work were done with the stellar evolutionary code LPCODE - see Althaus et al. (2005) and references therein. This code was previously used for studying the formation and evolution of H-deficient white dwarfs (Althaus et al. 2005; Miller Bertolami \& Althaus 2006) and EHB stars (Miller Bertolami et al. 2008). The code is based on a detailed description of the main physical processes involved in the formation of white dwarfs through late thermal pulses, particularly non-instantaneous mixing - see Althaus et al. (Alt05) for details. The standard mixing length theory for convection (with the free parameter $\alpha=1.6$ ) has been adopted. With regard to the microphysics considered in the present calculations, we employed radiative opacities from OPAL for arbitrary metallicity in the range from 0 to 0.1 (Iglesias \& Rogers 1996), supplemented at low temperatures with the Alexander \& Ferguson (1996) molecular opacities. Neutrino emission rates for pair, photo, and bremsstrahlung processes were taken from Itoh et al. (1996). For 
plasma processes we included the treatment presented in Haft et al. (1994). Conductive opacities are from Cassisi et al. (2007), which covers the whole regime where electron conduction is relevant. For the white dwarf regime we employed an updated version of the Magni \& Mazzitelli (1979) equation of state. The nuclear network takes into account 16 elements and 34 nuclear reactions for pp chains, $\mathrm{CNO}$ bi-cycle, helium burning, and carbon ignition and are detailed in Althaus et al. (Alt05).

All our sequences were computed in a self-consistent way, including the evolution of the chemical abundance distribution caused by element diffusion processes and burning during the whole white dwarf stage. It is worth mentioning that the opacity is also calculated self-consistently using the predictions of diffusion for the heavy element composition. In particular, for the case in which diffusion is considered, metallicity is taken as twice the abundances of $\mathrm{CNO}$ elements. We mention that time-dependent diffusion due to gravitational settling and chemical and thermal diffusion of nuclear species has been fully taken into account following the multicomponent gas treatment of Burgers (1969). The following species were considered: ${ }^{1} \mathrm{H}$, ${ }^{3} \mathrm{He},{ }^{4} \mathrm{He},{ }^{12} \mathrm{C},{ }^{14} \mathrm{~N}$, and ${ }^{16} \mathrm{O}$. The resulting flow equations for these species were integrated by using a semi-implict, finitedifference scheme. Details are given in Althaus et al. (2001a, 2005). Abundance changes were computed according to element diffusion and then to nuclear reactions and convective mixing. This detailed treatment of abundance changes by different processes during the white dwarf regime constitutes a key aspect in the evaluation of the importance of residual nuclear burning for the cooling of low-mass white dwarfs.

\subsection{Initial models}

The existence of a population of single He-core white dwarfs is not yet discarded (nor confirmed). Recent theoretical evidence based on energetic considerations suggests that these white dwarfs may be descendants of single supersolar metallicity progenitors with initial mass $M \leq 1 M_{\odot}$ (Meng et al. 2008). To derive starting configurations for our more massive He-core cooling sequences consistent with the evolutionary history of the progenitor star, we therefore simply removed mass from a $1 M_{\odot}$ model at the appropriate stages of its evolution (Iben \& Tutukov 1986; Driebe et al. 1998). Specifically, we forced the progenitor star to depart from the red giant phase just before the occurrence of the helium core flash. This is done for the three metallicities considered here. The resulting final stellar masses for each metallicity are listed in Table 1 with the total amount of hydrogen contained in the envelope, $M_{\mathrm{H}}$, and the surface abundance of hydrogen at maximum $T_{\text {eff }}$ at the beginning of the cooling branch. It is important to mention at this point that, as far as the evolutionary properties of our He-core white dwarfs are concerned, a detailed knowledge of the progenitor evolution, in particular the initial mass, is not a relevant issue (Driebe et al. 1998).

A look at Table 1 shows that the higher the mass of the remnant, the lower the residual mass of the hydrogen in the outer layers, similar to what is found for post-AGB remnants. As shown later, this hydrogen envelope will be responsible for residual hydrogen burning, thus markedly lengthening the cooling times. In this sense, the values of $M_{\mathrm{H}}$ listed in Table 1 can be considered as an upper limit to the expected evolutionary hydrogen envelope with which a given He-core white dwarf enters its cooling track. We also note from Table 1 that the values of $M_{\mathrm{H}}$ depend on the metallicity of the progenitor star. Specifically, $M_{\mathrm{H}}$ decreases as the progenitor metallicity increases
(Castellani et al. 1994). Because of this, the values of $M_{\mathrm{H}}$ derived here are somewhat lower than those quoted by Driebe et al. (1998) in their Table 2 for solar metallicity progenitors. Finally, for stellar masses below $M \approx 0.25 M_{\odot}$, mass loss during the RGB evolution has uncovered layers where hydrogen burning took place in prior stages, giving rise to pre-white dwarfs with He-enriched outer layers.

We applied the same procedure to obtain starting configurations in the case of He-core white dwarfs with lower masses. These white dwarfs are probably the result of mass transfer in close binary systems (Sarna et al. 2000), since exceedingly high ages would be needed to produce very low-mass white dwarfs through single-star evolution. However, we have not modeled the binary evolution leading to the formation of these stars, as done in Panei et al. (2007). It is worth mentioning in this context that, after the termination of the mass-loss episodes, the subsequent evolution of the model does not depend on the details of how most of the envelope was lost. In particular, the mechanical and thermal structure of the models are consistent with the predictions of binary evolution calculations (Driebe et al. 1998).

Finally, for He-core white dwarfs with masses higher than the canonical value of the core mass at the helium flash we artificially suppressed the core helium flash at the tip of the RGB. In this way, we derived He-core sequences of stellar masses of 0.513 and $0.522 M_{\odot}$. Whether or not this is a realistic procedure deserves further study, but this is beyond the scope of this paper. We mention that because of numerical difficulties, white dwarf models with higher He cores were computed only for the case of $Z=0.03$.

\subsection{Model atmosphere}

For all the evolutionary sequences in which diffusion has been considered, we computed accurate colors and magnitudes on the basis of improved LTE model atmospheres. The numerical code used is a new and updated version of the one described in Rohrmann et al. (2002). Models were computed assuming hydrostatic and radiative-convective equilibrium. Convective transport present in the cooler atmospheres was treated within the usual mixing-length approximation. The microphysics included in the model atmospheres comprises non-ideal effects in the gas equation of state and chemical equilibrium based on the occupation probability formalism as described in Rohrmann et al. (2002). The code includes $\mathrm{H}, \mathrm{H}_{2}, \mathrm{H}^{+}, \mathrm{H}^{-}, \mathrm{H}_{2}^{+}, \mathrm{H}_{3}^{+}, \mathrm{He}, \mathrm{He}^{-}$, $\mathrm{He}^{+}, \mathrm{He}^{2+}, \mathrm{He}_{2}^{+}, \mathrm{HeH}^{+}$, and $\mathrm{e}^{-}$. The level occupation probabilities are self-consistently incorporated in the calculation of the line and continuum opacities. Collision-induced absorptions due to $\mathrm{H}_{2}-\mathrm{H}_{2}, \mathrm{H}_{2}-\mathrm{He}$, and $\mathrm{H}-\mathrm{He}$ pairs are also taken into account (Rohrmann et al. 2002).

For the purpose of the present work, the model atmospheres explicitly include Ly $\alpha$ quasi-molecular opacity according to the approximation used by Kowalski \& Saumon (2006). Quasimolecular absorption results from perturbations of hydrogen atoms by interactions with other particles, mainly $\mathrm{H}$ and $\mathrm{H}_{2}$. Here, we consider extreme pressure-broadening of the line transition $\mathrm{H}(n=1) \rightarrow \mathrm{H}(n=2)$ due to $\mathrm{H}-\mathrm{H}$ and $\mathrm{H}-\mathrm{H}_{2}$ collisions, with the red wing extending far into the optical region. A detailed description of these collisional line-broadening evaluations will be presented in a forthcoming paper (Rohrmann et al. 2009). On the basis of the approximations outlined in Kowalski \& Saumon (2006), we evaluate the red wing absorption within the quasi-static approach using theoretical molecular potentials to describe the interaction between the radiator and the perturber. 
We also consider the variation in the electric-dipole transition moment with the interparticle distance. The $\mathrm{H}_{3}$ energy-potential surfaces contributing to collisions $\mathrm{H}-\mathrm{H}_{2}$ were taken from the analytical representations of Varandas et al. (1987), and the dipole transition moments were calculated from Petsalakis et al. (1988). Broadening of $\mathrm{Ly} \alpha$ line by $\mathrm{H}-\mathrm{H}$ collisions plays a minor role compared to $\mathrm{H}-\mathrm{H}_{2}$ encounters. The potential interactions for $\mathrm{H}-\mathrm{H}$ configurations were taken from Kolos \& Wolniewicz (1965) and the transition probability was assumed constant in this case. The main effect of the Ly $\alpha$ quasi-molecular opacity is a reduction of the predicted flux at wavelength smaller than $5000 \AA$ for white dwarfs cooler than $T_{\text {eff }} \approx 6000 \mathrm{~K}$.

\section{Evolutionary results}

In this section we describe the evolutionary properties of our sequences, placing strong emphasis on the role of residual nuclear burning during the white dwarf stage. We explore the implications of element diffusion and investigate the evolution of He-core white dwarfs with stellar masses higher than the canonical value of the helium core mass at the helium flash.

\subsection{Evolutionary sequences without element diffusion}

We begin by examining the results corresponding to the approximation usually adopted in most studies of these stars in which diffusion is not considered. As discussed previously, even though chemical diffusion operating at the base of the H-rich envelope appears to be an attractive hypothesis to match the pulsar spindown ages and the low-mass white dwarf cooling ages (Althaus et al. 2001a), its validity is still not well established, since diffusion could be mitigated and even suppressed by other physical processes, like strong magnetic fields. Exploring this possibility is a difficult task that would carry us too far afield. However, we believe it worthwhile to explore the extreme situation in which the evolution of He-core white dwarfs is not affected by diffusion processes. In fact, the evolutionary sequences computed disregarding diffusion allow an upper limit to the role of residual hydrogen burning to be established.

We concentrate on the sequences with $M=0.44524$ and $0.30154 M_{\odot}$ with progenitors of metallicity $Z=0.05$. We refer to these sequences as the standard sequences. Specifically, these sequences were calculated neglecting both gravitational settling and chemical diffusion and assuming a metallicity of $Z=0$ during the white dwarf regime. The Hertzsprung-Russell diagram for our selected standard sequences is displayed in Fig. 1. The absence of hydrogen shell flashes in the selected standard sequences is an expected feature and agrees with previous studies of low-mass white dwarfs. We find that hydrogen shell burning becomes unstable only for stellar masses below $\approx 0.26 M_{\odot}$.

We find that residual hydrogen burning via the pp chain reactions considerably slows down the cooling rate of evolved He-core white dwarfs resulting from metal-rich progenitors, in a similar way to what has been reported for low-mass He-core white dwarfs stemming from progenitors with less metal content (Castellani et al. 1994; Driebe et al. 1998; Sarna et al. 2000). The importance of residual nuclear burning as a source of energy is made clear in Fig. 2, which shows the temporal evolution of the hydrogen burning luminosity and the surface luminosity for the 0.44524 and $0.30154 M_{\odot}$ sequences. We have chosen to show the results corresponding to the case in which a $Z=0.05$ progenitor was adopted. Only the evolution on the final cooling phase is depicted in the figure. Residual hydrogen burning - for the

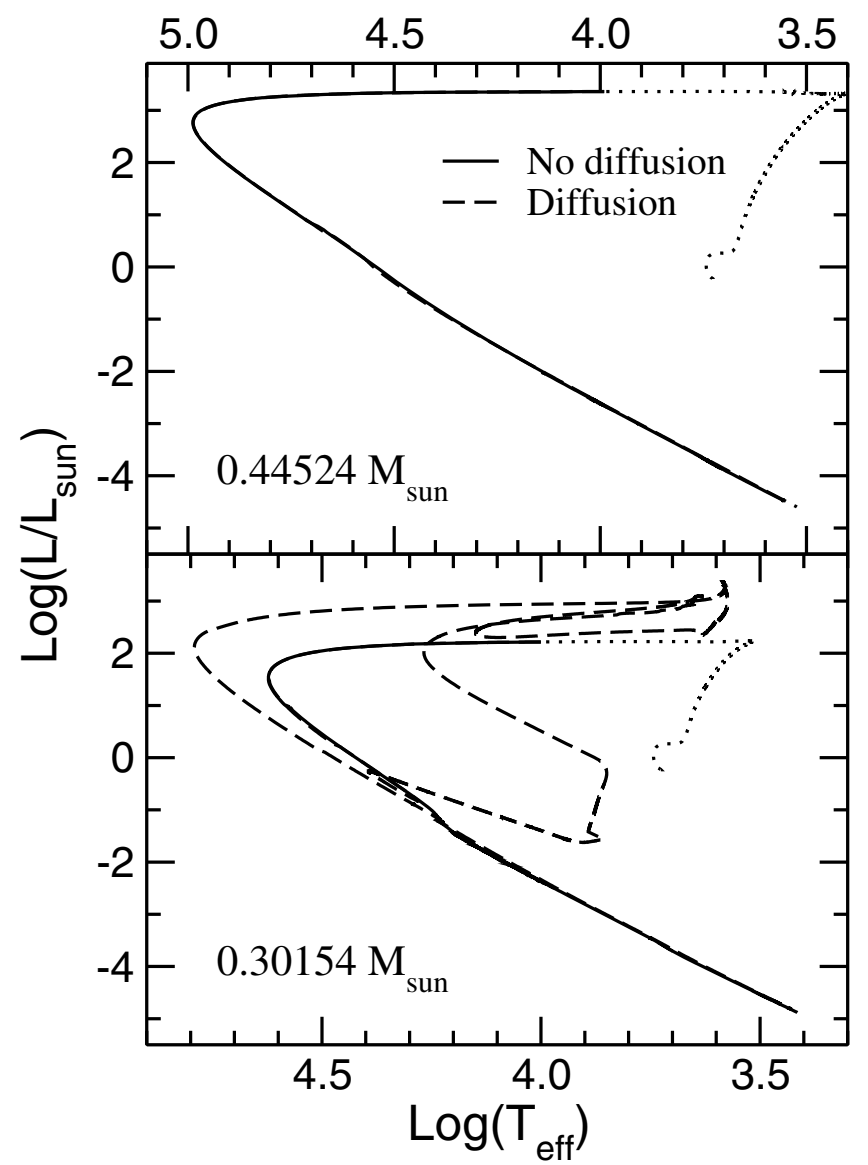

Fig. 1. Hertzsprung-Russell diagram for selected He-core white dwarf evolutionary sequences obtained from a $Z=0.05$ progenitor star. Dashed (solid) lines correspond to the case when element diffusion is (not) taken into account. The dotted lines show the evolution of the $1 M_{\odot}$ progenitor star.

stages shown in this figure the pp chain reactions are dominant appreciably contributes to the surface luminosity of the star even at very advanced stages of evolution. This is so despite the mass of the hydrogen retained in the surface layers at the beginning of the cooling phase being markedly smaller in metal-rich precursor stars. The contribution of residual nuclear burning to the energy budget of the star becomes more and more dominant as the stellar mass is decreased. We find that nuclear burning is also dominant at advanced evolutionary stages of He-core sequences that experience hydrogen shell flash episodes, i.e., for stellar masses below $\approx 0.26 M_{\odot}$. Finally, we find that the contribution of residual nuclear burning increases as the metallicity of the progenitor star decreases (see below).

The impact of residual nuclear burning on the evolution of He-core white dwarfs becomes clear by inspecting Fig. 3, which displays the temporal evolution of the surface luminosity for selected He-core sequences resulting from progenitors with metallicities $Z=0.05$ and $Z=0.03$. This figure shows the results of the full evolutionary calculations along with the situation when hydrogen burning is artificially suppressed. Residual hydrogen burning considerably influences the age of the white dwarf. Indeed, the evolution is delayed to very long ages by the active hydrogen burning zone. Clearly, when nuclear burning is neglected a substantial underestimate of the cooling age of the white dwarf is obtained. The magnitude of the delays introduced by nuclear burning is greater for decreasing stellar masses and metallicities. In most cases, evolution is 


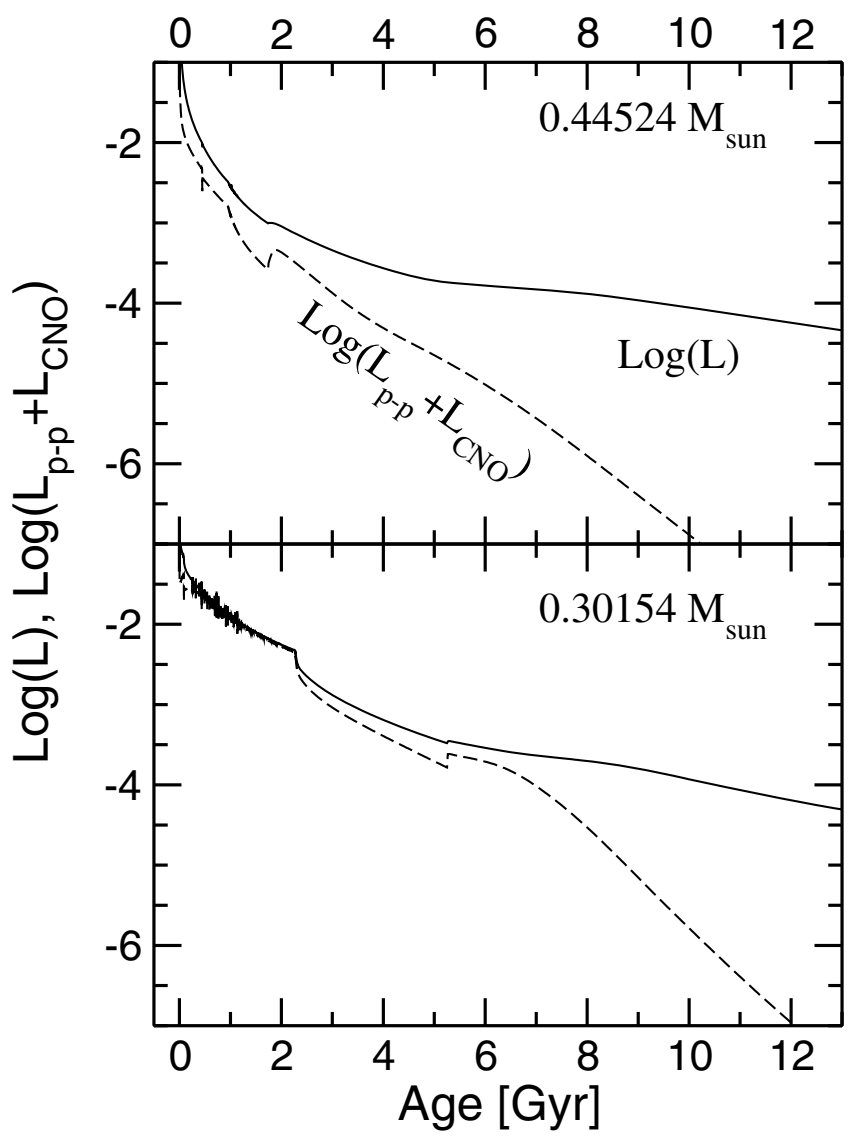

Fig. 2. Surface luminosity (solid lines) and nuclear luminosity due to hydrogen burning (dashed lines) as a function of age for two selected He-core cooling sequences. The panels are labeled with the corresponding stellar mass. The sequences correspond to the case in which we consider $Z=0.05$ progenitors. Diffusion processes are not considered.

essentially dictated by nuclear burning, giving rise to very long cooling ages. For the $0.44524 M_{\odot}$ sequence with $Z=0.05$, the magnitude of the delays amounts to 0.4 and $2.7 \mathrm{Gyr}$ at $\log \left(L / L_{\odot}\right)=-3$ and -4 , respectively. These delays amount to 2.2 and $5.3 \mathrm{Gyr}$ for the $0.30154 M_{\odot}$ sequence at the same luminosities. The effect of the metallicity is also quite apparent, increasing the cooling ages. For instance, for $Z=0.03$, the delays in the cooling times amount to 0.8 (4.6) and 3.5 (7.3) Gyr for the $0.45219(0.30316) M_{\odot}$ cooling sequences at the same luminosities. In summary, it is clear that the evolution of He-core white dwarfs resulting from progenitors stars with supersolar metallicity is considerably affected by nuclear burning. This is also true in the case of massive He-core white dwarfs. In particular, residual pp hydrogen burning strongly slows the evolutionary rate down to very low luminosities, a fact that must be considered when attempts are made to date metal-rich stellar populations using these stars.

\subsection{The effect of element diffusion}

We find that diffusion processes acting during the white dwarf cooling track strongly modify the shape of the chemical profiles, causing hydrogen to float to the surface, and helium and heavier elements to sink down, as expected. As a result, the pre-white dwarf with an initially $\mathrm{H}$ - and He-rich envelope turns into an object with a pure hydrogen envelope, that is, a white dwarf of the spectral type DA. We also find that the tail of the innermost hydrogen distribution chemically diffuses inwards into hotter layers as evolution proceeds along the cooling track. At high effective temperatures, this effect favors CNO thermonuclear flashes. These diffusion-induced hydrogen flashes are responsible for the hydrogen envelope with which the white dwarf enters into its final cooling track becoming markedly thinner than in the case when diffusion is neglected. These results are very similar to those found by Althaus et al. (2001a,b) and Panei et al. (2007) about the role that element diffusion plays in inducing additional thermonuclear flashes and about its responsibility for the occurrence of thin H-rich envelopes and a fast evolution at late stages of the evolution of He-core white dwarfs with solar metallicity progenitors. The results presented here show that the same occurs for He-core white dwarfs resulting from metal-rich progenitors. It is also worth noting that the occurrence of diffusion modifies the threshold for the occurrence of hydrogen shell flashes. In the case of He-core white dwarfs with $Z=0.05$ progenitors, we find that this threshold is about $0.37 M_{\odot}$, while all the sequences exhibit flashes for solar metallicity progenitors. The He-core cooling sequences with stellar masses lower than this do experience thermonuclear flashes (see Fig. 1 for the case of the $0.30154 M_{\odot}$ sequence). Because of the greater abundance of hydrogen carried by chemical diffusion into the burning region, this threshold value becomes larger for decreasing metallicities of the parent star.

As mentioned, as a result of diffusion-induced flashes, no residual nuclear burning is expected during the final cooling branch. This is made clear in Fig. 4 which displays the temporal evolution of the hydrogen-burning and surface luminosities during the final cooling branch for the same stellar masses shown in Fig. 2. The inclusion of diffusion predicts that residual hydrogen burning is a minor contribution to the star luminosity. In the case of the $0.44542 M_{\odot}$ sequence, which does not suffer from hydrogen flashes, a large fraction of the hydrogen content is burnt during the hot stages of the evolution. Thus, also in the case of sequences that do not suffer from hydrogen flashes, the white dwarf is ultimately left with a thin hydrogen-rich envelope, when diffusion is considered.

We expect the evolutionary timescales of He-core white dwarfs to be considerably affected by the inclusion of diffusion. This is borne out in Fig. 5, which clearly shows the impact of diffusion on the temporal evolution of the surface luminosity for the two stellar masses we analyzed previously. The evolutionary ages are substantially less when diffusion is considered. This reduction is more noticeable for lower metallicities of the parent star and stellar masses. The differences in cooling times resulting from the inclusion of diffusion are displayed in Fig. 7 for the $0.30154 M_{\odot}$ sequence. The delay in cooling times can reach up to $5 \mathrm{Gyr}$ (because of residual nuclear burning) if diffusion is not considered. We conclude that diffusion prevents hydrogen burning from being a main source of energy for most of the evolution of He-core white dwarfs with metal-rich progenitors. As a result, white dwarfs obtain energy from its relic thermal content, and the cooling ages become notably less when compared with those obtained in the case in which diffusion is neglected. Indeed, as shown previously, when diffusion is not considered, residual hydrogen burning after thermonuclear flashes is the dominant energy source even at advanced stages of evolution.

Finally, it should be noted that there is a dependence of the cooling times on the thickness of the hydrogen-rich envelope. To assess this effect, we computed the evolution of the $0.30316 M_{\odot}$ sequence for two different situations. In particular, in Fig. 6 we show the sequence corresponding to the case of a thick hydrogen envelope. This sequence was derived by 


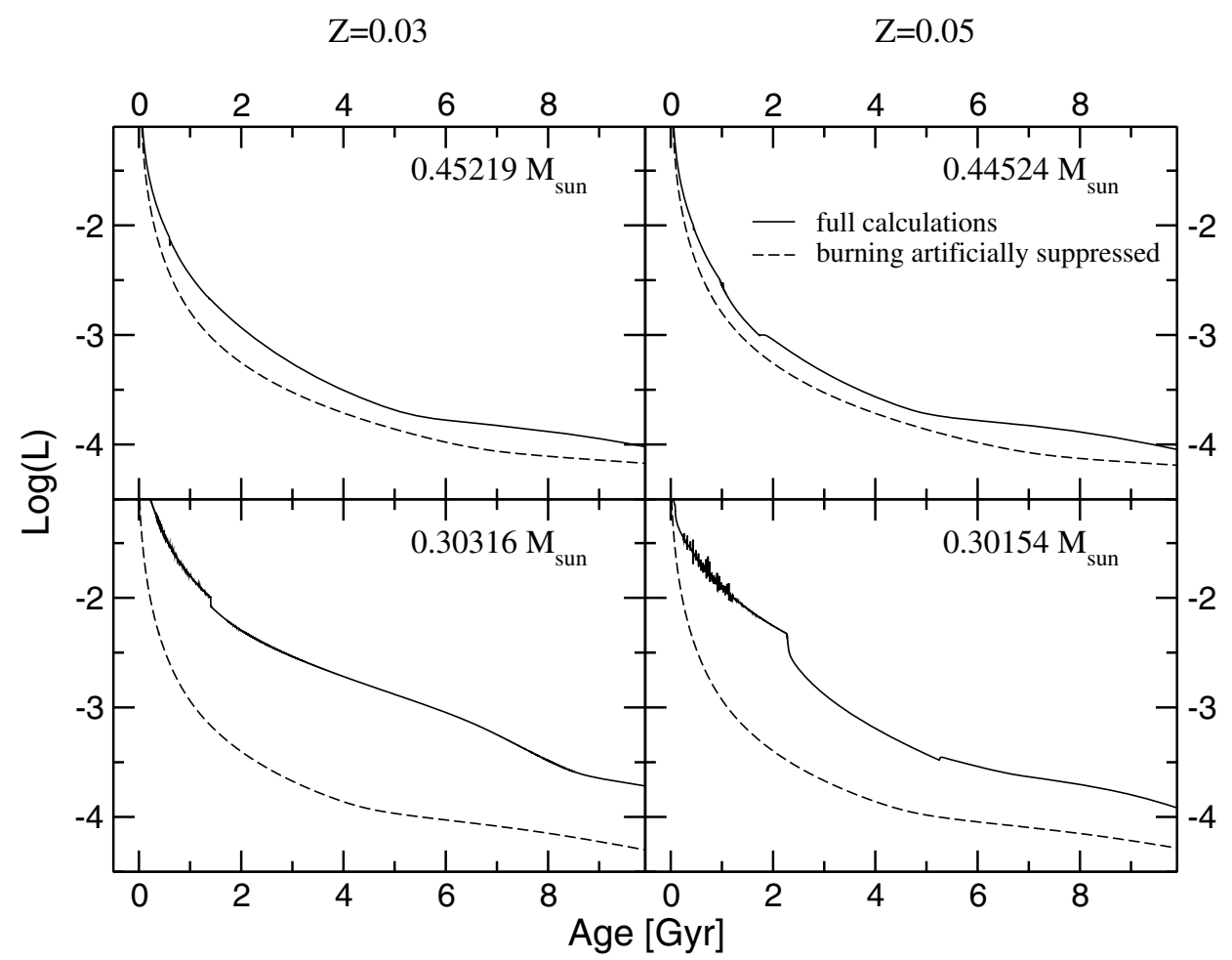

Fig. 3. Surface luminosity as a function of age for selected He-core sequences. Each panel is labeled with the corresponding stellar mass. Panels are also labeled with the corresponding metallicity of the progenitor stars. The solid lines show the results of the self-consistent calculations, while the dashed lines display the evolution when hydrogen burning is artificially suppressed. Element diffusion is not considered.

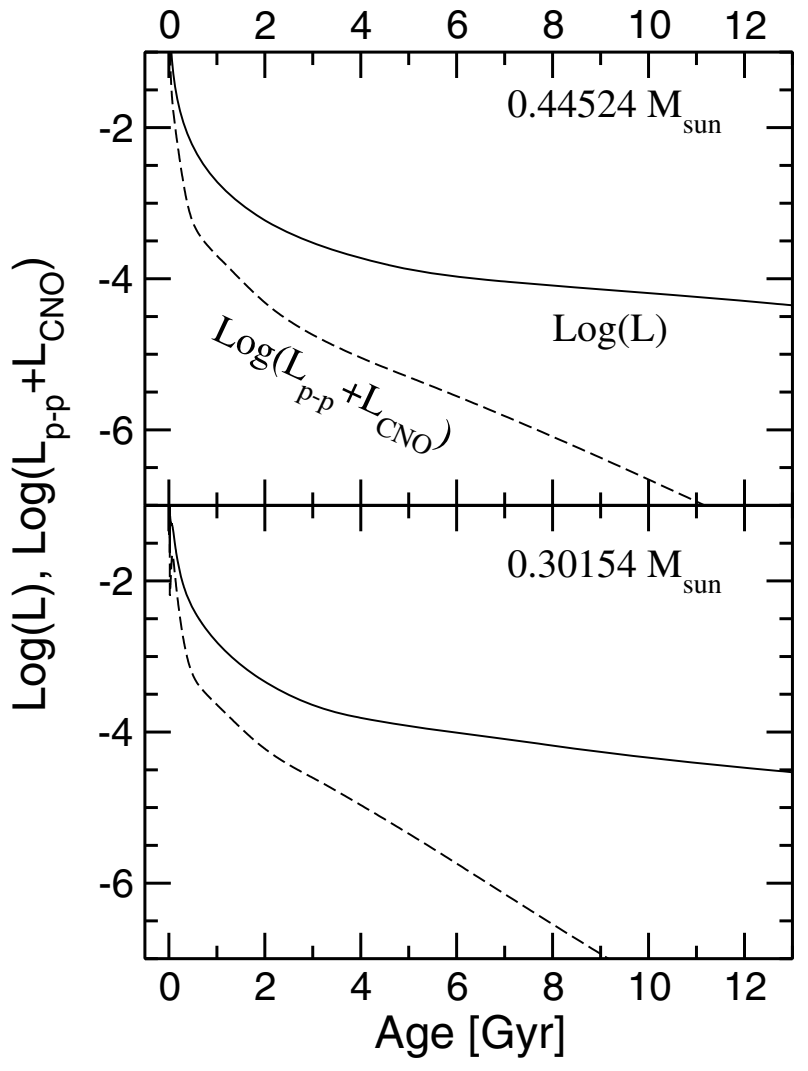

Fig. 4. Same as Fig. 2 but for the case in which diffusion processes are fully taken into account.

artificially suppressing the nuclear burning, thus avoiding the flash and formation of a thin envelope. The sequence depicted with a dashed line illustrates the situation for the case of a thin envelope that results from the hydrogen flash. Both sequences, though not entirely self-consistent with the assumptions of the modeling considered in this work, allow us to isolate the opacity effects from the nuclear energy effects. Although the cooling times do show a non-negligible dependence on the mass of the residual hydrogen envelope, we emphasize that the effect of the opacity resulting from a different layering (Tassoul et al. 1990) is much weaker than the effects resulting from residual hydrogen burning.

\subsection{Massive helium-core white dwarfs}

The possibility that some He-core white dwarfs in NGC 6791 could have higher masses than the canonical value of the core mass at the helium flash $\left(\approx 0.45 M_{\odot}\right.$ for metal-rich, low-mass RGB stars) has been recently raised by Bedin et al. (2008b) to explain some observed features of the RGB and of the cooling sequence of NGC 6791. We have extended the scope of the present work by computing evolutionary sequences for He-core white dwarfs with masses higher than $0.45 M_{\odot}$. To this end, as explained in Sect. 3.2, we artificially suppressed the core helium flash at the tip of the RGB. In this way, we derived He-core sequences of stellar masses of 0.513 and $0.522 M_{\odot}$.

Evolutionary results for these massive white dwarfs are summarized in Fig. 8, which displays the surface luminosity as a function of age for an He-core sequence of $0.5211 M_{\odot}$ with a $Z=0.03$ progenitor. The solid line shows the case in which diffusion is considered, while the dashed line illustrates the case in which diffusion is neglected. Following the trend we find for the case of the more massive He-core white dwarfs discussed previously, it is clear that the age of massive He-core white dwarfs is not markedly influenced by diffusion processes. This is an 


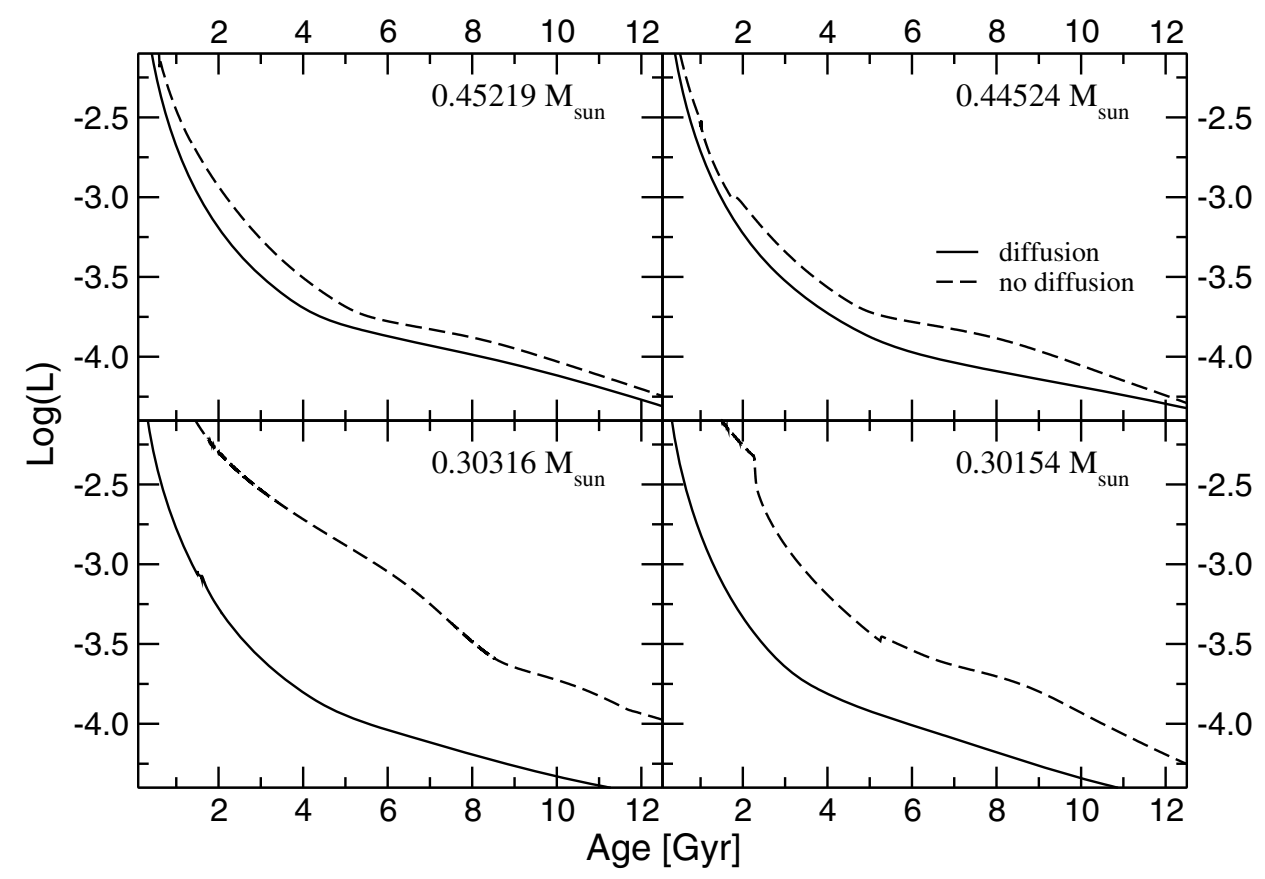

Fig. 5. Surface luminosity as a function of age for selected He-core sequences as labeled by their stellar mass for $Z=0.05$ and $Z=0.03$ progenitors, right and left panels respectively. Solid (dashed) lines correspond to the situation when element diffusion is (not) considered.

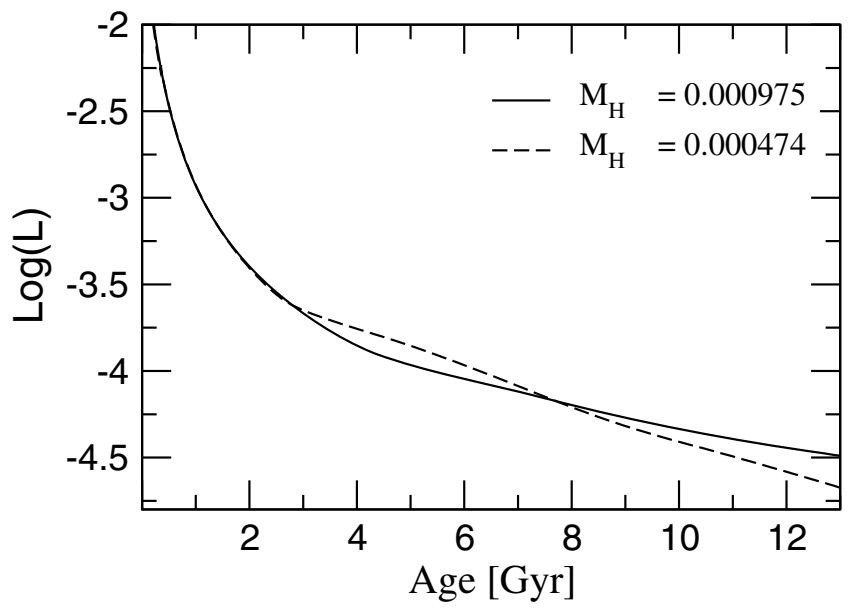

Fig. 6. Surface luminosity as a function of age for our He-core white dwarf sequence of $0.30316 M_{\odot}$ derived from a $Z=0.03$ progenitor and with different hydrogen envelope masses. In both cases, element diffusion has been considered and residual nuclear burning has been artificially suppressed. The mass of the hydrogen envelopes is given in solar masses.

expected result, because for such massive He-core white dwarfs, the H-rich envelope left after RGB evolution is substantially smaller in this case, see Table 1 , with the consequence that residual nuclear burning is not a major source of energy even in the case that element diffusion is neglected. This behavior contrasts with the situation we find in less massive He-core white dwarfs, for which, when diffusion is neglected, nuclear burning constitutes the main energy source even at advanced stages, delaying their evolution to very long ages.

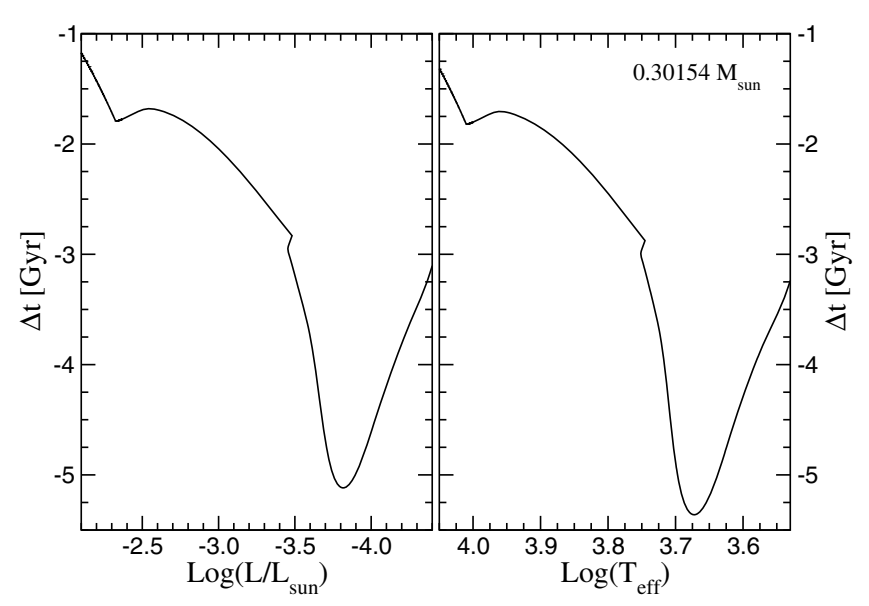

Fig. 7. Differences in cooling times (in Gyr) resulting from the inclusion of diffusion corresponding to the sequence with $0.30154 M_{\odot}$ derived from a $Z=0.05$ progenitor.

\section{Colors and magnitudes}

For all our cooling sequences in which diffusion has been considered, we computed accurate colors and magnitudes based on new and improved non-gray model atmospheres that explicitly include Ly $\alpha$ quasi-molecular opacity according to the approximation used by Kowalski \& Saumon (2006). The calculations were done for a pure hydrogen composition and for the HST ACS filters (Vega-mag system) and UBVRI photometry. As an example of these calculations, we show in Figs. 9 and 10 selected color-color and color-magnitude diagrams for all our sequences with $Z=0.03$ progenitors. In both diagrams, all our sequences exhibit the well known turn-off in their colors at very low effective temperature and become blue with further evolution. This is a result of collision-induced absorption from 


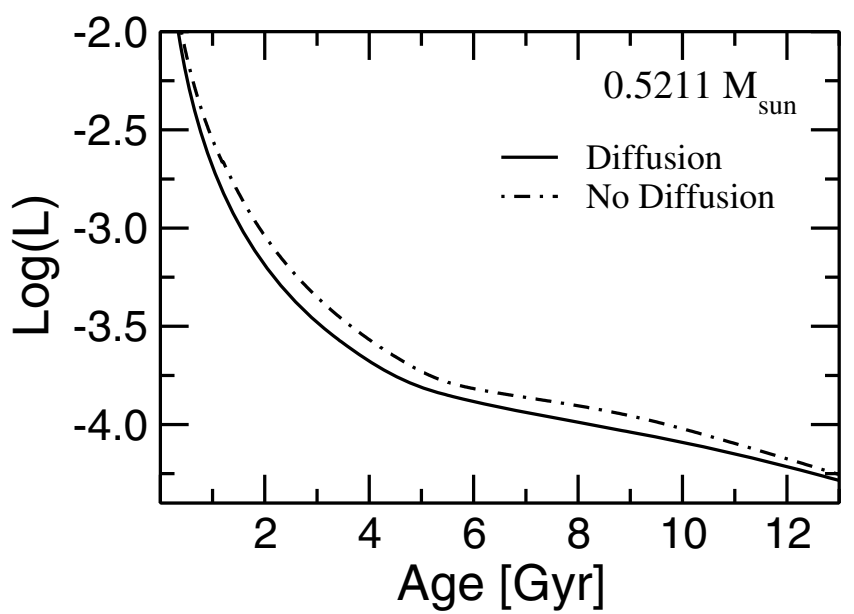

Fig. 8. Surface luminosity as a function of age for our massive He-core white dwarf sequence of $0.5211 M_{\odot}$ derived from a $Z=0.03$ progenitors with artificially suppressed core helium flash. The solid (dashed) line corresponds to the situation in which element diffusion is (not) considered.

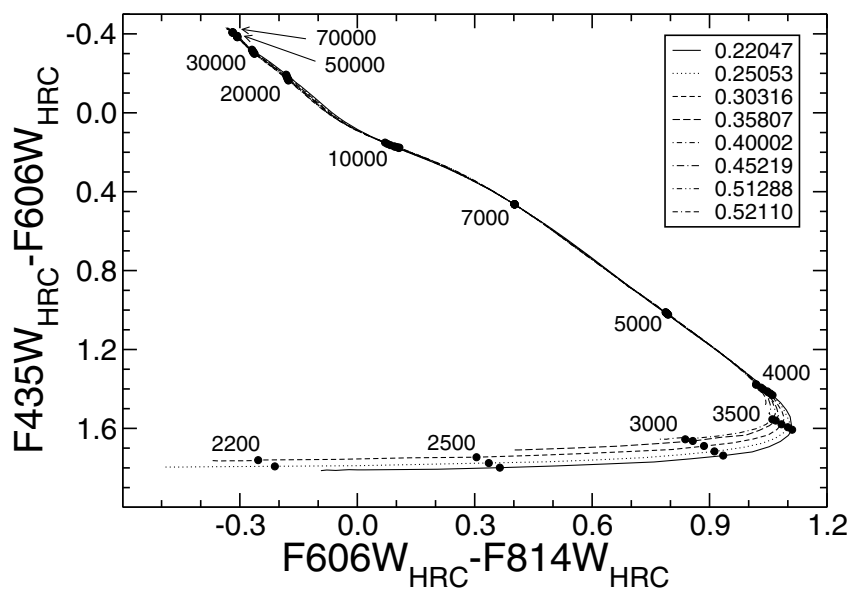

Fig. 9. $F 435 W-F 606 W, F 606 W-F 814 W$ color-color diagram for our He-core white dwarf sequences with $Z=0.03$ progenitors. Pure hydrogen composition is assumed. Selected $T_{\text {eff }}$ values are labeled along the curves.

molecular hydrogen, a process that reduces the infrared flux and forces radiation to emerge at higher frequencies. Finally, we mention that the inclusion of $\operatorname{Ly} \alpha$ quasi-molecular opacity makes the sequences redder, thus delaying the turn to the blue (particularly in the UBVRI photometric system, which we do not show here for the sake of conciseness). This is because the collisional effect on $\operatorname{Ly} \alpha$ reduces the predicted flux at wavelengths shorter than $5000 \AA$ for cool white dwarfs.

\section{Discussion and conclusions}

In this paper we have studied the evolution of He-core white dwarfs resulting from metal-rich progenitors. The absence of detailed calculations for such white dwarfs, as well as the discovery of He-core white dwarfs in the metal-rich cluster NGC 6791, prompted us to compute a detailed grid of evolutionary sequences for He-core white dwarfs appropriate for the study of these stars in metal-rich clusters. Specifically, we computed a total of 30 evolutionary sequences that cover a wide range of stellar masses. Three supersolar metallicities for the progenitor star were considered: $Z=0.03,0.04$, and 0.05 . In addition, to

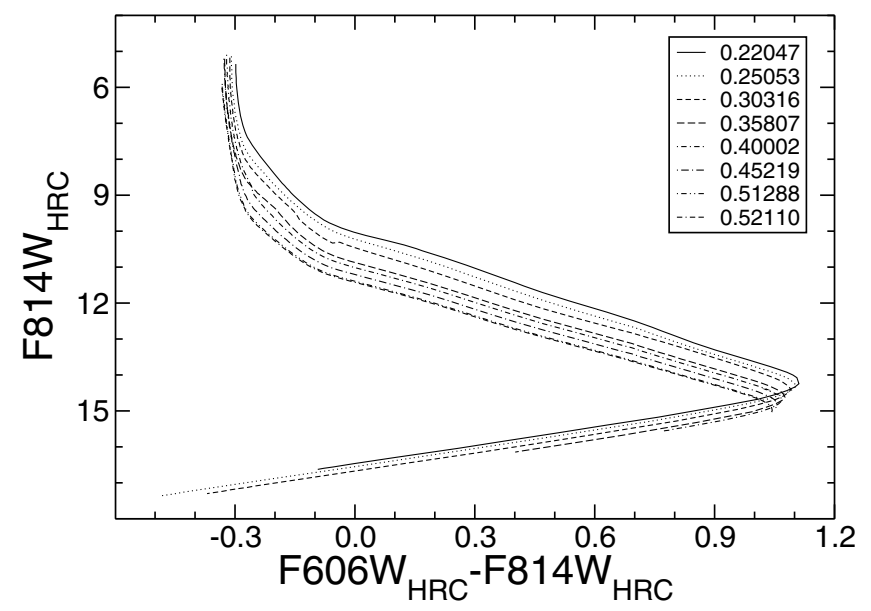

Fig. 10. $F 814 W, F 606 W-F 814 W$ color-magnitude diagram for our Hecore white dwarf sequences with $Z=0.03$ progenitors. Pure hydrogen composition is assumed.

study the evolution of He-core white dwarfs with masses higher than the He core mass at the He flash we also computed additional sequences by artificially suppressing the core He flash at the tip of the RGB. The evolution of all white dwarfs was followed from the end of mass-loss episodes during the pre-white dwarf evolution down to very low surface luminosities.

Our computations capture the bulk of the essential microphysics involved in the evolution of low-mass white dwarfs particularly time-dependent diffusion and hydrogen burning - as well as the evolutionary history of progenitor stars formed in metal-rich environments. These features allowed us to provide a detailed account of the evolutionary properties of these white dwarfs. The paper was also intended to explore the role of element diffusion and residual nuclear burning in the late evolution of He-core white dwarfs having supersolar metallicity. In addition to the evolutionary calculations, we computed for all our sequences accurate colors and magnitudes based on new and improved non-gray model atmospheres that explicitly include Ly $\alpha$ quasi-molecular opacity according to the approximation used by Kowalski \& Saumon (2006). The grid of He-core sequences presented here, together with that presented in Serenelli et al. (2002) for He-core white dwarfs of low-metallicity progenitors, constitutes a solid and consistent frame for interpretating observations of He-core white dwarfs in stellar clusters of different metallicities.

We find that residual hydrogen burning strongly influences the late stages of white dwarf cooling, substantially delaying the evolution by several Gyr in some cases. This is so despite the mass of hydrogen retained in the surface layers at the beginning of the cooling stage being markedly lower in the case of metal-rich precursor stars. The contribution of residual nuclear burning to the energy budget of the white dwarf becomes more dominant for lower stellar masses. We arrive at the opposite conclusion if element diffusion is allowed to operate. Indeed, diffusion processes substantially impact the evolution of He-core white dwarfs. The reason for this is that an important fraction of the hydrogen content left in the white dwarf is burnt during the early stages of evolution by diffusion-induced hydrogen burning, with the consequence that the star is left with almost no nuclear energy at late stages. Thus, diffusion strongly mitigates the role of residual nuclear burning, making the evolution rely solely on the thermal content stored in the ions, as assumed in some 
previous studies of low-mass white dwarfs (Althaus \& Benvenuto 1997; Hansen \& Phinney 1998).

In view of the importance of diffusion for the late stages of the evolution of He-core white dwarfs, it is very important to establish that diffusion occurrs in these stars. It can be argued that the role of element diffusion could by mitigated and even suppressed by processes like turbulence or magnetic fields. In this regard, we find that a reduction of the diffusion velocity by a factor of $\sim 0.7$ is enough to inhibit the diffusion-induced hydrogen shell flashes that are responsible for the fast evolution at late stages. However, there are numerous and different pieces of evidence that diffusion is an important process that occurs both in the surface layers and in the deep interior of white dwarf stars. The purity of the atmosphere of most white dwarfs and the observed spectral evolution are best understood in terms of diffusion. The existence of some cool white dwarfs with helium atmospheres and traces of carbon (DQs) are explained in terms of the outward diffusion of the inner carbon profile (Pelletier et al. 1986). For low-mass white dwarfs with helium cores, element diffusion also appears to be a viable process able to explain the extremely fast evolution required to match the white dwarf age with the spin-down ages of some millisecond pulsars and, thus, in explaining the observed age dichotomy (Panei et al. 2008; Althaus et al. 2001a). In a different context, asteroseismology can in principle provide important information about the internal stratification of white dwarfs. For instance, for intermediatemass white dwarfs it has been argued (Metcalfe et al. 2005) that diffusion occurs in the deep envelope of the pulsating He-rich white dwarf GD 358. All this evidence hints at the occurrence of diffusion processes in the deep interior of white dwarfs and give strong support to our result that residual pp burning in He-core white dwarfs is not expected to be an important source of energy for significant periods of time. In closing, we emphasize that detailed tabulations of our evolutionary sequences and colors for both the HST ACS filters and UBVRI photometric system can be found at our web site http: //www . fcaglp. unlp. edu.ar/ evolgroup or can be obtained upon request from the authors.

Acknowledgements. We acknowledge the suggestions and comments of our referee, D. Winget, which substantially improved the original version of this paper. This research was supported by AGENCIA: Programa de Modernización Tecnológica BID 1728/OC-AR, by the AGAUR, by MCINN grant AYA2008-04211-C02-01, by the European Union FEDER funds and by PIP 6521 grant from CONICET. L.G.A. also acknowledges a PIV grant of the AGAUR of the Generalitat de Catalunya. Finally, we thank R. Martinez and H. Viturro for technical support.

\section{References}

Althaus, L. G., \& Benvenuto, O. G. 1997, ApJ, 477, 313

Althaus, L. G., Serenelli, A. M., \& Benvenuto, O. G. 2001a, MNRAS, 323, 471

Althaus, L. G., Serenelli, A. M., \& Benvenuto, O. G. 2001b, ApJ, 554, 1110
Althaus, L. G., Serenelli, A. M., Panei, J. A., et al. 2005, A\&A, 435, 631 Bassa, C. G. 2006, Ph.D. Thesis, Universiteit Utrecht

Bassa, C. G., van Kerkwijk, M. H., \& Kulkarni, S. R. 2003, A\&A, 403, 1067

Bedin, L. R., Salaris, M., Piotto, G., et al. 2008a, ApJ, 679, L29

Bedin, L. R., King, I. R., Anderson, J., et al. 2008b, ApJ, 678, 1279

Burgers, J. M. 1969, Flow Equations for Composite Gases (New York: Academic Press)

Calamida, A., Corsi, C. E., Bono, G., et al. 2008, ApJ, 673, L29

Casagrande, L., Flynn, C., Portinari, L., Girardi, L., \& Jimenez, R. 2007, MNRAS, 382, 1516

Cassisi, S., Pothekin, A. Y., Pietrinferni, A., Catelan, M., \& Salaris, M. 2007, ApJ, 661, 1094

Castellani, V., Luridiana, V., \& Romaniello, M. 1994, ApJ, 428, 633

Castellani, V., Calamida, A., Bono, G., et al. 2007, ApJ, 663, 1021

Cool, A. M., Grindlay J. E., Cohn, H. N., Lugger, P. M., \& Baylyn, C. D. 1998, ApJ, 508, L75

Driebe, T., Schönberner, D., Blöcker, T., \& Herwig, F. 1998, A\&A, 339, 123

Edmonds, P. D., Gilliland, R. L., Heinke, C. O., Grindlay, J. E., \& Camilo, F. 2001, ApJ, 557, L57

Flynn, C. 2004, PASA, 21, 126

Haft, M., Raffelt, G., \& Weiss, A. 1994, ApJ, 425, 222

Hansen, B. M. S. 2005, ApJ, 635, 522

Hansen, B. M. S., \& Phinney, E. S. 1998, MNRAS, 294, 557

Iben, I. Jr., \& Tutukov, A. V. 1986, ApJ, 311, 742

Iglesias, C. A., \& Rogers, F. J. 1996, ApJ, 464, 943

Itoh, N., Hayashi, H., Nishikawa, A., \& Kohyama, Y. 1996, ApJS, 102, 41

Kalirai, J. S., Bergeron, P., Hansen, B. M. S., et al. 2007, ApJ, 671, 748

Kepler, S. O., Kleinman, S. J., Nitta, A., et al. 2007, MNRAS, 375, 1315

Kippenhahn, R., Kohl, K., \& Weigert, A. 1967, Z. Ap., 66, 58

Kolos, W., \& Wolniewicz, L. 1965, J. Chem. Phys., 43, 2429

Kowalski, P. M., \& Saumon, D. 2006, ApJ, 651, L137

Landsman, W., Aparicio, J., Bergeron, P., Di Stefano, R., \& Stecher, T. P. 1997, ApJ, 481, L93

Magni, G., \& Mazzitelli, I. 1979, A\&A, 72, 134

Meng, X., Chen, X., \& Han, Z. 2008, A\&A, 487, 625

Miller Bertolami, M. M., \& Althaus, L. G. 2006, A\&A, 454, 845

Miller Bertolami, M. M., Althaus, L. G., Unglaub, K., \& Weiss, A. 2008, A\&A, 491,253

Moehler, S., \& Bono, G. 2008, in White dwarfs, ed. R. Napiwotzki, \& M. Burleigh, in press [arXiv: 0806.4456$]$

Nelson, L. A., Dubeau, E., \& MacCannell, K. A. 2004, ApJ, 616, 1124

Origlia, L., Valenti, E., Rich, R. M., \& Ferraro, F. R. 2006, ApJ, 646, 499

Panei, J. A., Althaus, L. G., Chen, X., \& Han, Z. 2007, MNRAS, 382, 779

Pelletier, C., Fontaine, G., Wesemael, F., Michaud, G., \& Wegner, G. 1986, ApJ, 307, 242

Petsalakis, I. D., Theodorakopoulos, G., \& Wright, J. S. 1988, J. Chem. Phys., 89,6850

Rohrmann, R. D., Serenelli, A. M., Althaus, L. G., \& Benvenuto, O. G. 2002, MNRAS, 335, 499

Sarna, M., Ergma, E., \& Gerškevitš-Antipova, J. 2000, MNRAS, 316, 84

Serenelli, A. M., Althaus, L. G., Rohrmann, R. D., \& Benvenuto, O. G. 2002, MNRAS, 337, 1091

Tassoul, M., Fontaine, G., \& Winget, D. E. 1990, ApJS, 72, 335

Taylor, J. M., Grindlay, J. E., Edmonds, P. D., \& Cool, A. M. 2001, ApJ, 553, L169

van Kerkwijk, M. H., Bassa, C. G., Jacoby, B. A., \& Jonker, P. G. 2005, in Binary

Radio Pulsars, ed. F. A. Rasio, \& I. H. Stairs, ASP, 328, 357

van Loon, J. T., Boyer, M. L., \& McDonald, I. 2008, ApJ, 680, L49

Varandas, A. J. C., Brown, F. B., Mead, C. A., Truhlar, D. G., \& Blais, N. C. 1987, J. Chem. Phys., 86, 6258

Webbink, R. F. 1975, MNRAS, 171, 555

Winget, D. E., \& Kepler, S. O. 2008, ARA\&A, 46, 157 